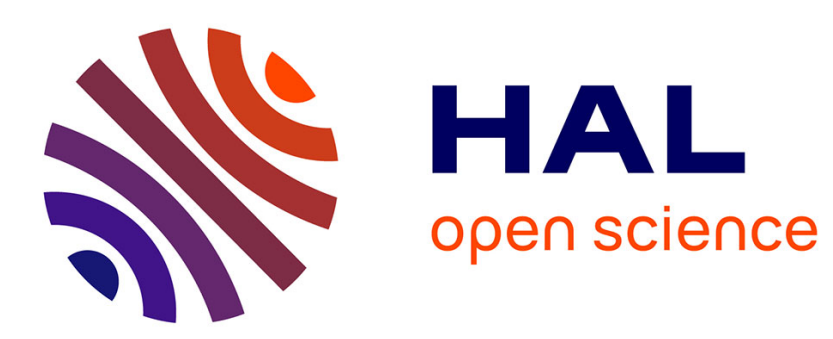

\title{
Considérations épistémologiques sur les cercles vicieux épigénétiques du vieillissement
}

\author{
Paul-Antoine Miquel
}

\section{To cite this version:}

Paul-Antoine Miquel. Considérations épistémologiques sur les cercles vicieux épigénétiques du vieillissement. Journal de la Société de Biologie, 2004, 198 (3), pp.293-298. halshs-00714967

\section{HAL Id: halshs-00714967 https://shs.hal.science/halshs-00714967}

Submitted on 11 Apr 2014

HAL is a multi-disciplinary open access archive for the deposit and dissemination of scientific research documents, whether they are published or not. The documents may come from teaching and research institutions in France or abroad, or from public or private research centers.
L'archive ouverte pluridisciplinaire HAL, est destinée au dépôt et à la diffusion de documents scientifiques de niveau recherche, publiés ou non, émanant des établissements d'enseignement et de recherche français ou étrangers, des laboratoires publics ou privés. 


\title{
Considérations épistémologiques sur les cercles vicieux épigénétiques du vieillissement
}

Journal de la Société de Biologie, 198(3), 2004.

Paul-Antoine Miquel

Université de Nice

\begin{abstract}
Ageing is not a disease. Age changes occur in every adult animal. It takes place in all species. Yet, malign tumours, and neurodegenerative diseases are growing with age. Nobody dies from aging. Death results from age-associated diseases. Muscular and digestive atrophies are rising with age. But can we say that digestive atrophy lead to a bad nutrition, or is it not the contrary? Where is the chicken and where is the egg? We are closed in a vicious circle.
\end{abstract}

A vicious circle is not a simple cycle. It's an irreversible phenomenon that happens when a system of objects is acting on itself. Vicious circles are coming too at the molecular level. This paper tries to show that the destruction of elastin and of fibronectine in connective tissues produces a self destruction effect. Von Neumann showed that we can imagine a model of self reproduction with the help of reproductive automata. We attempts to show that nature has imagined a model to produce self destruction, with the use of degradation of fibronectine and elastin in peptides.

This self destruction process is neither purely stochastic, nor genetically programmed. It's an epigenetical process. It's not present at the beginning, like an a priori instruction, it is emerging. Then we try to give a specific unusual philosophical definition to the word "emergence." 


\section{Résumé en français}

Le vieillissement n'est pas une maladie. Il atteint tout animal dépassant un certain âge et il concerne presque toutes les espèces vivantes. Pourtant les tumeurs malignes, l'arthrose et les maladies neurodégénératives augmentent avec l'âge. On ne meurt pas de vieillesse. On meurt de poly-pathologies. Alors, où est la poule, où est l'œuf ? Où est le vieillissement, où est la maladie?

Le vieillissement, ce sont les atrophies musculaires et digestives. Mais est-ce l'atrophie digestive qui provoque la dénutrition ou la dénutrition qui la produit ? Il y a des cercles vicieux du vieillissement. Le cercle vicieux n'est pas un cycle. C'est un phénomène irréversible qui est propre à un système d'objets agissant sur lui-même.

On se souvient que les tissus parenchymateux peuvent régénérer leurs cellules. Mais ce mécanisme fonctionne de moins en moins bien. Les fibres élastiques et fonctionnelles sont peu à peu remplacées par des tissus conjonctifs. N'est-il pas surprenant alors de retrouver, au cœur de cette dynamique de destruction de la fibronectine et de l'élastine des cercles vicieux moléculaires?

Nous défendons ici une double hypothèse. Scientifiquement, tout d'abord, nous souhaitons montrer que ces processus d'autodestruction ne sont ni stochastiques, ni génétiques. Ils sont épigénétiques.

Philosophiquement ensuite, nous les considérons comme des processus émergents. Un système d'objets qui agit sur lui-même n'est pas pourvu d'une âme ou d'un esprit frappeur. Il est simplement décrit par le fait que son action complète sa description. C'est cela qui détermine le sens que nous donnons au concept d'émergence.

L'autodestruction n'est pas là au commencement. Elle n'est pas programmée génétiquement. Elle résulte de la destruction de certaines molécules et des effets que cette destruction entraîne. Pourtant cette résultante est autre chose qu'une simple déduction. Et cette biologique du vieillissement est autre chose qu'une simple logique. 


\section{Introduction}

L'analyse du processus du vieillissement a commencé depuis les travaux de Harman ou de Hayflick à descendre au niveau moléculaire. Les biologistes et les biochimistes utilisent le terme de cercle vicieux pour désigner plusieurs types de processus post-traductionnels.

Ainsi, par exemple, les radicaux libres libérés par le processus de respiration cellulaire agissent non seulement sur les enzymes responsables du transport des électrons, mais aussi sur le génome des mitochondries, notamment au niveau des gènes responsables de la synthèse. Comme les mitochondries ne disposent pas de mécanismes de réparation de l'ADN, les mutations génétiques induites finissent par provoquer des effets négatifs sur le processus de respiration cellulaire conduisant à la fabrication de davantage de radicaux libres (J. Miquel, 1998).

Nous voulons concentrer notre attention dans cet article sur un autre exemple de cercle vicieux épigénétique et post-traductionnel. L'augmentation graduelle de la synthèse d'élastine dans les tissus conjonctifs engendre pour des raisons biochimiques sa dégradation en peptides eux-mêmes reconnus par un récepteur induisant une amplification de la dégradation de l'élastine par la production d'élastases plus ou moins puissantes et de décharges radicalaires (L. Robert, 1997).

Nous entendrons par épigénétique en un sens spécifique le fait que ce processus circulaire ne peut pas être commandé par l'action des gènes, mais qu'il est produit par l'action de ce processus sur lui-même. C'est l'action de ce processus sur lui-même qui est responsable de l'émergence d'une nouvelle fonction biologique, une fonction d'autodestruction et modifie donc ainsi sa description initiale. Von Neumann (1996) a montré naguère qu'il ne fallait pas confondre reproduction et autoreproduction en faisant émerger la fonction d'auto-reproduction à partir de l'action concertée d'automates reproducteurs. Nous voulons indiquer ici d'une manière analogue qu'il ne faut pas confondre destruction et auto-destruction. Le vieillissement moléculaire que nous allons analyser à travers le fonctionnement de ces processus consiste en l'émergence d'une fonction d'auto-destruction. Telle est le première thèse que nous défendrons.

La seconde est que le langage de la logique formelle traditionnelle ne nous permet pas d'apprécier à sa juste mesure philosophique cette bio-logique du vieillissement moléculaire. 
La logique formelle traditionnelle en effet s'interdit d'abord, en vertu du principe du cercle vicieux, la formulation de proposition ou d'énoncés auto-référentiels et contradictoires. Mais elle ne permet pas non plus de décrire les conditions susceptible de faire de la fonction d'autodestruction une propriété émergente.

\section{1- Le Principe du cercle vicieux en logique}

a-Le principe du cercle vicieux (PCV) est énoncé simultanément, quoique de manière différente par Russell et Poincaré au début du vingtième siècle. Russell, contrairement à Poincaré et aux constructivistes, reconnaît l'existence de l'infini actuel et donc des classes finies et infinies (D. Vernant, 1993). Le principe du cercle vicieux s'applique d'abord aux classes, puis aux fonctions propositionnelles et enfin aux propositions.

L'origine de ce principe est à chercher dans l'énoncé du célèbre paradoxe des classes de Russell qui va inaugurer la célèbre crise des fondements en logique et en mathématiques qu'il expose pour la première fois dans une lettre à Frege le 16 juin 1902. Certaines classes sont éléments d'elles-mêmes. Soit A la classe de toutes les classes C qui ne se contiennent pas elles-mêmes comme éléments :

(1) $(\mathrm{C})[(\mathrm{C}$ appartient à $\mathrm{A}) \equiv(\mathrm{C}$ n'appartient pas à $\mathrm{C})]$

Mais A est une classe comme les autres. On peut donc écrire que A est un C. Par substitution alors on a :

(2) [A appartient à $\mathrm{A} \equiv \mathrm{A}$ n'appartient pas à $\mathrm{A}]$

Nous pouvons caractériser alors le cercle vicieux comme une formule auto-référentielle (selfreference) et auto-réflexive (reflexiveness) qui engendre une contradiction (D.Vernant, 1993).

On trouve également ce paradoxe au niveau de la fonction propositionnelle. Si on définit intensionnellement $\mathrm{fx}^{*}$ la fonction propositionnelle, par opposition avec la totalité $\mathrm{fx}$ des valeurs qu'elle peut prendre, il est impossible d'écrire $\mathrm{f}(\mathrm{fx} *)$, car $\mathrm{fx} *$ ne peut être une valeur de la fonction f (B. Russell, 1989). 
Russell pense alors qu'il est possible d'éviter les cercles vicieux par ce qu'il appelle la théorie des types. Le type est l'ensemble des arguments possibles qui satisfont une fonction propositionnelle. Si nous écrivons par exemple «x est un homme», «Socrate » est un argument qui satisfait la proposition « $\mathrm{x}$ est un homme ». Une fonction propositionnelle de type 1 ne peut avoir comme valeurs possibles que des objets ou individus et non des fonctions. Aucune fonction ne peut en effet se prendre elle-même pour objet. Autrement nous courrons le risque de tomber sur des paradoxes.

b- Poincaré soutenait que les cercles vicieux naissaient de l'obligation de penser des classes infinies. Mais on peut aussi trouver des cercles vicieux dans la proposition elle-même. Si au lieu d'écrire « Socrate est un menteur », nous écrivons « je mens », nous tombons sur un nouveau cercle vicieux célèbre. Le traitement de ce cercle vicieux dans le langage plus contemporain du logicien Tarski (1972) serait le suivant :

(1) « $\mathrm{i} »: \mathrm{i}$ est fausse

Si on applique le critère de vérité de Tarski, on a

(2) «i est fausse » est vraie ssi i est fausse

Si nous admettons que la signification de (1) est que l'on peut remplacer $i$ est fausse par $\ll i »$, nous avons

(3) « $\mathrm{i} »$ est vraie ssi i est fausse

D'où vient le cercle vicieux alors ? D'une double erreur : d'abord, une proposition entre guillemets ne peut pas se substituer à une proposition sans guillemets. L'une est un énoncé langagier, l'autre est un énoncé métalangagier.

Ensuite, on peut attribuer une valeur de vérité ou de fausseté à un énoncé langagier, mais du point de vue d'un langage sur cet énoncé langagier. La vérité et l'erreur sont des propriétés métalangagières. Aucune proposition ne peut donc s'attribuer à elle-même de la vérité ou de la fausseté.

Le cercle vicieux apparaît donc dans la proposition si celle-ci fait référence à ellemême dans son énoncé, et si le fait qu'elle fasse référence à elle-même lui donne une forme 
contradictoire. On est donc tenté de conclure que tout énoncé autoréférentiel contradictoire est un énoncé mal formé. Pour éviter la contradiction, il semble suffire de faire en sorte que nous ne formulions plus d'énoncés autoréférentiels. C'est vers cette direction que semblent nous engager Russell avec sa théorie des types et Tarski, avec sa théorie des hiérarchies de langage.

Cette conclusion nous oriente dans une direction réformatrice. Nos langues naturelles regorgent en effet d'énoncés autoréférentiels susceptibles de déboucher sur des cercles vicieux, comme le montre le paradoxe d'Eubulide ou le paradoxe du Menteur. Cela semble être le signe qu'elles sont impropres à construire des propositions bien formées. Cette orientation de pensée sera celle de la philosophie analytique anglo-saxonne dans le début du vingtième Siècle, sous l'impulsion de Russell et de Carnap. Pour elle, la plupart des problèmes épistémologiques ou plus généralement philosophiques ne sont que des problèmes de logique résultant de l'utilisation d'énoncés mal formés.

La question que nous posons est donc la suivante : peut-on également utiliser la logique pour résoudre des faux problèmes épistémologiques en biologie?

\section{Les cercles moléculaires dans les tissus conjonctifs}

Peut-on supposer d'abord que le vieillissement est programmé génétiquement, comme pourrait l'être également le développement ? Le terme de «programme génétique du vieillissement » a longtemps fait partie de la littérature (R. Russell,1987). On a opposé les tenants de la théorie du stress oxydatif, comme Harman, à ceux de la théorie de la programmation génétique. On a aussi tenté de réconcilier les deux théories.

Mais qu'entend on par programmation génétique ? Le langage qu'on emploie n'est pas si loin de celui de la logique formelle. On peut considérer que les algorithmes de ce programme sont comme des fonctions propositionnelles $\mathrm{P}$ et que les données traitées par ce programme sont comme les variables définissant l'ensemble des valeurs possibles que ces fonctions propositionnelles peuvent prendre (d). Le processus de vieillissement résulte alors du traitement de ces données par un programme : $\mathrm{P}(\mathrm{d})$. Ce langage permet de comparer le traitement des données par un programme à l'étude des propriétés d'une fonction propositionnelle. Encore faut-il, cependant, que l'on puisse décrire ces données et définir le traitement de ces données par ce programme. Mais l'on voit également qu'une très forte ambiguïté est contenue dans cette expression. On peut entendre d'abord par programmation 
génétique le simple fait que les gènes codent pour la synthèse de protéines, au sens où il y a ainsi une information génétique stockée dans les gènes déterminant cette synthèse. Mais l'idée que le vieillissement soit programmé renvoie plutôt à l'idée d'une information génétique déterminant un processus de transformation de l'organisme, impliquant la synthèse de protéines de structure et de protéines régulatrice à partir d'un ensemble de gènes. Il y aurait ainsi, comme une fonction de détérioration de l'organisme encodée dans son génome.

On entend à présent par tissus « conjonctifs », par opposition à «parenchymateux », la partie plus organisationnelle que fonctionnelle des tissus. Si l'on examine comment ils se forment, nous découvrons en effet que des gènes codent, à l'intérieur de chaque cellule pour la synthèse de protéines qui vont constituer la Matrice Extra Cellulaire (MEC). L'élastine est ainsi responsable de l'élasticité des tissus, le collagène de leur solidité. Parmi les glycoprotéines de structure, la fibronectine sert de colle moléculaire. Mais il y a aussi la laminine, etc. La matrice interagit avec le génome de chaque cellule et régule la traduction des gènes en protéines. Nous voyons déjà à ce niveau l'ambiguïté que nous évoquions précédemment. Ce n'est pas parce que le génome code pour la synthèse des protéines de la Matrice Extra Cellulaire, qu'il y a un gène qui détermine entièrement le processus de formation de cette Matrice. Il est au contraire épigénétique, puisqu'il résulte de l'interaction des composantes de la Matrice avec le génome des cellules qui la synthétise sous l'influence du milieu extérieur. Ainsi, par exemple, des tractions en rythme d'une lame élastique portant une culture de cellules musculaires lisses vasculaires provoquent une forte augmentation de la synthèse de l'élastine (L.Robert, 1995). Donc s'il y a un programme du vieillissement, il n'est pas situé au niveau des gènes.

Examinons maintenant de plus près le problème du vieillissement. C'est l'exemple même d'un processus intégré et multifactoriel. Nous pouvons l'expliquer moléculairement par le raccourcissement des télomères et l'inefficacité progressive de la télomérase (L. Hayflick, 1994). Mais certains auteurs vont jusqu'à nier aujourd'hui le rôle de la télomérase dans la limitation de la prolifération cellulaire.

On peut mettre en valeur l'action des radicaux libres. Mais on sait que certains organismes fabricant plus de radicaux libres (comme le pigeon) vieillissent plus longtemps que d'autres (comme le rat) qui en fabriquent moins. On sait que les radicaux libres ne jouent pas qu'un rôle négatif et qu'il y a une polarisation de leur activité à la fois positive et négative. R. Burdon (1996) met en valeur le rôle signalétique de l'eau oxygénée (H202). G. Faury ( 1998) 
a montré que l'oxyde nitrique (NO) produit par le couplage entre le récepteur de l'élastine et une NOsynthétase joue un rôle vasodilatateur sur les cellules épithéliales vasculaires du rat. Pourtant en présence de l'anion superoxyde, il peut engendrer un dérivé toxique (ONOO־). Il est vrai que certains gènes permettent d'expliquer comment, en désactivant la transcription de l'ADN ribosomal, l'organisme de la levure ou du nématode peuvent ralentir le métabolisme cellulaire par déacétylation des histones (L. Guarente, 2003). Mais le risque est grand là encore, d'extrapoler à l'ensemble du monde vivant des conclusions tirées à partir d'expérimentations faites sur des animaux modèles.

Concentrons nous donc sur un phénomène spécifique sans prétendre expliquer ainsi l'ensemble du processus du vieillissement. Il se caractérise chez le mammifère par une altération des fonctions spécifiques des organes et par le renforcement des travées conjonctives. C'est dire que la synthèse des molécules qui composent la MEC ne cesse d'augmenter.

Prenons l'exemple de l'élastine. En un premier temps nous pouvons dire en effet que la synthèse de l'élastine est programmée génétiquement. Nous désignons par là la relation d'irréversibilité entre l'action du gène de l'élastine et la synthèse de cette protéine qui ne peut agir en retour directement sur la nature de ce gène. Synthétiser l'élastine est en un sens la fonction accomplie par ce gène en vertu des informations qu'il reçoit.

Mais pour comprendre le processus de vieillissement ici, c'est à un niveau post-traductionnel qu'il faut se placer. Y a-t-il un gène qui détermine la transformation post-traductionnelle des tissus de l'organisme ? C'est alors que nous pourrions parler de vieillissement programmé. Qu'est-ce qui pourrait nous pousser à penser que ce n'est pas un gène ? Y a-t-il quelque chose ici comme un processus post traductionnel qui agit sur lui-même ? Il faudrait faire dans ce cas la différence entre ces deux énoncés :

\section{(1) Le processus post-traductionnel acquiert le pouvoir d'agir sur lui-même.}

\section{(2) Un gène détermine l'action du processus post traductionnel.}

Nous voyons en effet que le premier énoncé ressemble de très près à un énoncé autoréférentiel. Dans quelle mesure peut-on dire que ce processus acquiert le pouvoir d'agir sur lui-même? 


\section{Amplification de l'élastolyse et émergence de l'auto-destruction}

Elastine

Calcium et Lipides

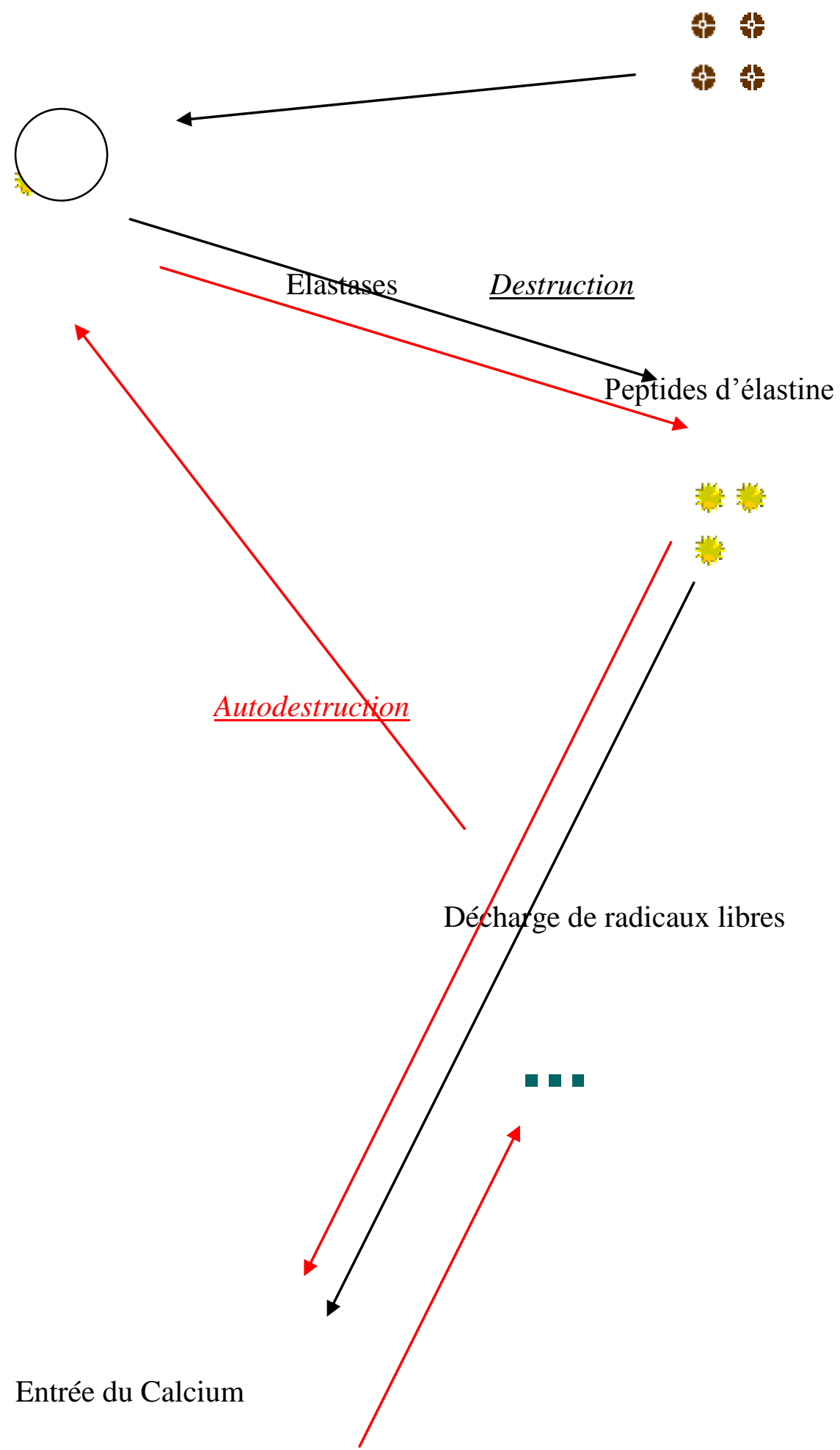

Récepteur de l'élastine-Laminine

Sortie

Entrée Calcium

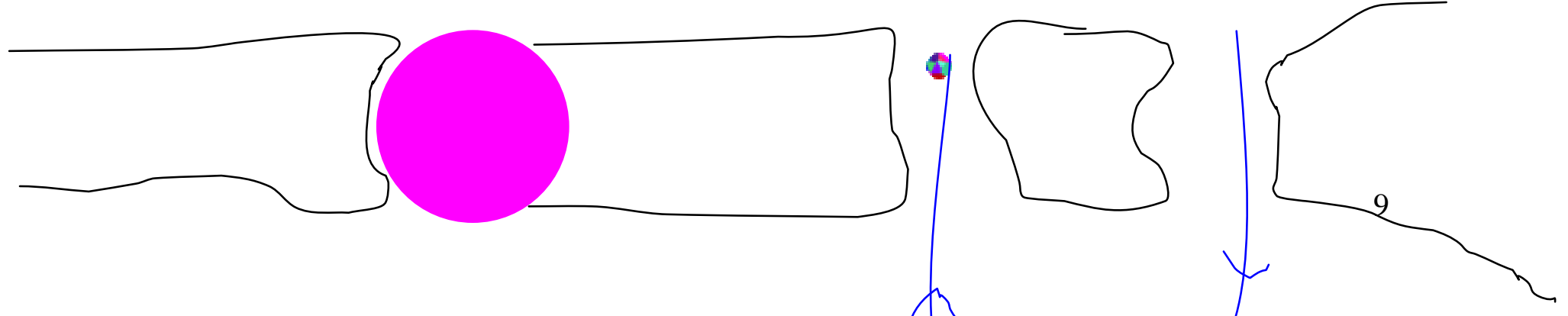


Appelons processus post-traductionnel cet ensemble d'éléments composé :

1- de la molécule d'élastine qui a pour fonction de donner de l'élasticité aux tissus.

2- de l'interaction de l'élastine avec le calcium et les lipides.

3- du fait que la synthèse d'élastine augmente au fur et à mesure du développement de l'individu.

4- du récepteur de l'élastine à la surface de la cellule.

Le fonctionnement de ce processus commence par la dégradation de l'élastine en peptides. Cette dégradation engage l'activation du récepteur qui déclenche la synthèse de protéases ainsi que des décharges de radicaux libres. Le processus est alors modifié par son fonctionnement d'une manière irréversible.

Dans ce processus des molécules d'élastine ont été détruites. Mais le fait nouveau est l'activation de la destruction de la molécule d'élastine par la destruction de la molécule d'élastine. Cette fonction d'activation de la destruction à partir de la destruction première est une propriété nouvelle de ce processus qui résulte de son action sur lui-même. Ce n'est plus une simple destruction, c'est une auto-destruction.

\section{Bio-logique moléculaire du vieillissement}

Le processus post-traductionnel ne peut pas être considéré comme une suite d'instructions, un algorithme à partir duquel une fonction est remplie en fonction des données qu'il reçoit, comme la fonction de destruction de l'élastine. D'abord, il faudrait admettre pour cela qu'il y a deux types d'algorithmes : ceux propres au programme génétique et ceux propres au processus post-traductionnel. Cela poserait déjà le difficile problème de leurs relations hiérarchiques. Mais ensuite cet étrange algorithme post-traductionnel en vient à ce traiter lui-même comme donnée, et à se modifier en fonction de la manière dont il se traite comme donnée. A partir de la destruction initiale de l'élastine, il s'assigne à lui-même une nouvelle fonction d'auto-destruction.

Il n'est donc pas question de vouloir résoudre les problèmes posés par la bio-logique du vivant en s'appuyant sur les consignes de la logique formelle traditionnelle. Mais pourquoi ? Nous entendions naguère par l'expression «jeu de la vie » le fait que de nouvelles 
fonctions puissent émerger dans l'organisme, sans que cette nouveauté constitue pourtant un mystère. Nous appelions émergence le fait que le fonctionnement d'un système naturel doive être incorporé dans sa description. Celle-ci ne peut plus alors être réduite à sa description initiale.

Tel est le cas ici de la fonction d'auto-destruction. Elle ne fait pas partie de la description initiale du processus épigénétique que nous avons analysé. Elle résulte du fonctionnement de ce processus. Ainsi le fait que ce processus puisse se traiter lui-même comme donnée résulte du fait que son fonctionnement entre dans sa description, ce que l'énoncé du principe du cercle vicieux dans le cadre de la logique formelle ne nous permettait pas directement de comprendre. Le cercle vicieux n'est pas un état caractéristique de ces processus épigénétiques. C'est plutôt un résultat.

\section{Conclusion}

Il n'est pas sûr d'abord que le langage logique puisse éliminer les énoncés autoréférentiels et les paradoxes qui en découlent en cherchant à les éviter. Ainsi dans l'exemple de la hiérarchie des langages de Tarski, où est le langage du logicien formulant la théorie de la hiérarchie des langages ? S'il fait partie de cette hiérarchie, alors il ne peut pas garantir la vérité de ses propres énoncés, et s'il peut le faire, il ne fait pas partie de la hiérarchie et construit des énoncés autoréférentiels.

Mais ce qui est sûr, c'est que la bio-logique du vivant en produit et qu'elle produit des énoncés auto-référentiels qui sont également contradictoires. Elle ne le fait pas par

dysfonctionnement, mais dans le cadre normal de son fonctionnement. Il y a une rationalité dans le fait que le vivant ait besoin de se détruire pour se construire par les mécanismes de l'apoptose, il y en a certainement également une dans le fait qu'il se détruit pour se détruire lorsque nous commençons à vieillir. C'est ainsi que, dans un nouveau contexte, sonne étrangement à nos oreilles l'injonction d'Henri Bergson : «contre toute logique » il nous faut admettre l'existence des cercles vicieux moléculaires.

Non seulement cette bio-logique produit de tels énoncés, mais elle produit les conditions qui permettent à ces énoncés d'émerger. Il ne faut pas concevoir ceux-ci comme des propriétés essentielles des systèmes biologiques, mais plutôt comme ce qui résulte du fait que leur fonctionnement fait partie de leur description. 
C'est ainsi que ces cercles bio-logiques obéissent à une stratégie de destruction, ils ne sont pas le simple constat d'une usure moléculaire, du désordre qui s'installe dans les machines. Ainsi seulement pouvons-nous comprendre que certains organismes végétaux mettent tant de temps à se détruire, voire ne se détruisent pas. Ainsi pouvons nous comprendre qu'il ne s'agit pas d'une simple destruction, mais d'un processus à travers lequel la destruction s'accélère elle-même. Le vivant ne s'abîme pas. Il fabrique en s'abîmant des bombes qui ont la propriété en explosant de fabriquer de nouvelles bombes encore plus puissantes que les premières.

\section{Références:}

Burdon R. H., 'Control of Cell Proliferation by Reactive Oxygen Species', Biochemical Society Transactions, Vol 24, 1996, pp1028-1031.

Hayflick L., Why and how we age, Balantine Books, NY, 1994.

Faury G. et alli, Proceeding National Academy of Science, 1998, 82, pp 328-36.

Guarente L., Ageless Quest, Cold Spring Harbor Laboratory Press, New York, 2003.

Miquel J., "An update of the oxygen stress-mitochondrial mutation theory of aging: genetic and evolutionary implications', Exp. Gerontology, 1998, 33, pp 113-126.

Robert L., 'Les Mécanismes épigénétiques du vieillissement', C.R. Société de Biologie, 1997, 191, pp. 247-52. Robert L., Le Vieillissement, faits et théories, Paris, Domino, Flammarion, 1995, p. 24.

B. Russell, Principia Mathematica, tr. J.M. Roy, Paris, PUF, 1989, p 275.

Russell R. L., 'Evidence For and Against the Theory of Developmentally Programmed Aging', Modern Biological Theories of Aging, H.R. Warner ed., N.Y., Raven Press, 1987.

Tarski A., Logique, sémantique, métamathématique, tr. fr. Granger, Paris, A. Colin, 1972, p. 164.

Vernant D., La philosophie mathématique de Russell, Paris, Vrin, 1993, §40-42.

Von Neumann J. Théorie générale et logique des automates, tr. fr. J.P. Auffrand, Paris, Champs, Vallon, 1996. 